view of special interest attached to observing conditions in England, apropos the advisability or otherwise of erecting large telescopes in Great Britain rather than in South Africa, it may be noted that the sky was completely unclouded on 41 nights only in the entire year.

Special interest is attached to the time determinations at Greenwich during the past year, as an international longitude programme was organised in October-November, 1933, in which Greenwich took an active part. A new type of chronograph, with a very light syphon pen and a tape running at $2 \cdot 5 \mathrm{~cm}$. per sec. instead of the old-fashioned barrel, has been installed. It is now estimated that the probable error to be attached to each determination of time is $0.010^{3}$, but that there are still personal equations to be attached to individual observers in spite of the use of the moving wire micrometer. Two of the routine observers differ from one another by $0.04 \mathrm{~s}$ At present the mean of three regular observers is taken as standard for Greenwich Mean Time. It is hoped to construct a personal equation machine in the future. Meanwhile, a cathode ray oscillograph has been purchased with which it is intended to investigate time lags in the reception of wireless time signals.
R. v.d.R.W.

\title{
Spectroscopically Pure Substances
}

$\mathrm{A}^{\mathrm{B} O}$ OUT ten years ago, Messrs. Adam Hilger, Ltd., the well-known firm of optical instrument makers, first undertook to place on the market substances of a high degree of purity such as could be relied upon for the exacting requirements of spectroscopic work. It was intended that such supplies should not only be the purest obtainable by modern technique, but should also be accompanied by a detailed report of the exact amounts of residual impurities as measured by both chemical and spectroscopic methods. In some cases, indeed, so pure have been the products obtained, that only quantitative spectroscopic analysis has been available. Messrs. Hilger have obtained their supplies from specialists in all parts of Europe and the United States, and they have been produced in the laboratories of universities, technical institutes, industrial works, and of private individuals, as well as from the National Physical Laboratory.

Up to 1932, metals alone had been produced, but recently oxides and salts have been added to the list. of these highly purified materials some twenty-two are metals, eleven are rare earths, and the remainder are largely commoner salts and oxides. The magnitude of this achievement and the considerable advance in the technique of both preparation and analysis made in recent years may perhaps best be judged from the facts relating to a few typical substances.

Manganese. This metal has been prepared in the National Physical Laboratory according to the formula of Dr. M. V. Gayler. A high-frequency induction furnace is used to distil the metal at a temperature just above its melting point $\left(1244^{\circ} \pm 3^{\circ} \mathrm{C}\right.$.), at a pressure of one or two $\mathrm{mm}$. Brittle silver-grey nuggets obtained in this way were found to have a purity of 99.99 per cent. The principle impurities were aluminium $(0.0003$ per cent), iron $(0.0024$ per cent), nitrogen $(0.0027$ per cent) and phosphorus $(0.0007$ per cent).

Germanium has been prepared in the laboratories of the Sir John Cass Technical Institute of at least 99.98 per cent purity. The impurities found were iron (less than 0.001 per cent) and sulphur $(0.002$ per cent).

Cadmium and Zinc. Both of these are of exceptional purity, namely, of more than 99.999 per cent. The zinc contains copper (less than 0.0001 per cent), lead (about 0.0002 per cent), and slight traces of calcium and iron. The cadmium contains traces of bismuth, lead and copper, in each case to an extent of less than one part in a million.

Columbium of $99 \cdot 8$ per cent purity has been prepared. According to Dr. W. F. Meggers the metal is free from the frequently associated elements vanadium, tantalum, zirconium and molybdenum and contains as impurity chiefly tin-which is not of great inconvenience from a spectroscopic point of view.

Iron rods obtained electrolytically with a purity of 99.96 per cent are available. These contain 0.02 per cent of non-metals (of no spectroscopic interest), silicon $(0.01$ per cent) and 0.01 per cent of various metals of which full details are supplied.

Silver with a purity of 99.999 per cent is probably one of the purest substances produced. It has been freed entirely from copper and contains as residual impurity chiefly calcium.

Gallium with a purity of 99.938 per cent, which contains only 0.05 per cent of zinc, is probably the purest specimen of this element so far produced.

Copper rods of 99.964 per cent purity have been obtained. Of the impurities, oxygen accounts for 0.03 per cent, and the remainder is constituted of various metals in very small amounts. Very recently, Messrs. Hilger have obtained supplies of copper of which the impurities are only about one twentieth those quoted above.

We turn now to a few chemical compounds which are made available under the trade name of 'Specpure' substances.

Beryllium Oxide and Chloride of 99.99 per cent purity contain only iron $(0.005$ per cent) with a trace of sodium and a minute trace of magnesium. These compounds have been hitherto very rare in a highly purified form.

Calcium Chloride is notable for its high general purity of 99.993 per cent and especially for its complete freedom from strontium. The latter achievement must be rare, if not unique, since Hönigschmid's recent atomic weight determination was made on material not quite free from strontium.

Similarly chlorides of strontium, aluminium and cobalt, and also powdered silica, all of 99.99 per cent purity, can be supplied. Lead nitrate with a purity of 99.999 per cent and containing only traces of bismuth, copper and antimony, is also noteworthy.

These illustrations will suffice to show the excellence and range of the materials now available. A new standard of purity has been introduced on an exten. sive scale, and Messrs. Adam Hilger deserve the congratulations and thanks of physicists and chemists alike for their enterprise and its well-merited success. R. C. Johnson. 